\title{
On approximating the quasi-arithmetic mean
}

Tie-Hong Zhao', Bu-Chuan Zhou ${ }^{2}$, Miao-Kun Wang ${ }^{2}$ and Yu-Ming Chu ${ }^{2 *}$ (D)

"Correspondence:

chuyuming2005@126.com

2Department of Mathematics, Huzhou University, Huzhou, China

Full list of author information is

available at the end of the article

\section{Abstract}

In this article, we prove that the double inequalities

$$
\begin{aligned}
\alpha_{1} & {\left[\frac{7 C(a, b)}{16}+\frac{9 H(a, b)}{16}\right]+\left(1-\alpha_{1}\right)\left[\frac{3 A(a, b)}{4}+\frac{G(a, b)}{4}\right] } \\
& <E(a, b) \\
& <\beta_{1}\left[\frac{7 C(a, b)}{16}+\frac{9 H(a, b)}{16}\right]+\left(1-\beta_{1}\right)\left[\frac{3 A(a, b)}{4}+\frac{G(a, b)}{4}\right], \\
& \left.\frac{7 C(a, b)}{16}+\frac{9 H(a, b)}{16}\right]^{\alpha_{2}}\left[\frac{3 A(a, b)}{4}+\frac{G(a, b)}{4}\right]^{1-\alpha_{2}} \\
& <E(a, b) \\
& <\left[\frac{7 C(a, b)}{16}+\frac{9 H(a, b)}{16}\right]^{\beta_{2}}\left[\frac{3 A(a, b)}{4}+\frac{G(a, b)}{4}\right]^{1-\beta_{2}}
\end{aligned}
$$

hold for all $a, b>0$ with $a \neq b$ if and only if $\alpha_{1} \leq 3 / 16=0.1875, \beta_{1} \geq 64 / \pi^{2}-6=$ $0.484555 \ldots, \alpha_{2} \leq 3 / 16=0.1875$ and $\beta_{2} \geq(5 \log 2-\log 3-2 \log \pi) /(\log 7-\log 6)=$ $0.503817 \ldots$, where $E(a, b)=\left(\frac{2}{\pi} \int_{0}^{\pi / 2} \sqrt{a \cos ^{2} \theta+b \sin ^{2} \theta} d \theta\right)^{2}, H(a, b)=2 a b /(a+b)$, $G(a, b)=\sqrt{a b}, A(a, b)=(a+b) / 2$ and $C(a, b)=\left(a^{2}+b^{2}\right) /(a+b)$ are the quasi-arithmetic, harmonic, geometric, arithmetic and contra-harmonic means of $a$ and $b$, respectively.

MSC: 26E60; 33C05

Keywords: Quasi-arithmetic mean; Harmonic mean; Geometric mean; Arithmetic mean; Contra-harmonic mean

\section{Introduction}

Let $a, b>0, p:(0, \infty) \mapsto(0, \infty)$ be a strictly monotone real-valued function, $\theta \in(0,2 \pi)$ and

$$
r_{n}(\theta)= \begin{cases}\left(a^{n} \cos ^{2} \theta+b^{n} \sin ^{2} \theta\right)^{1 / n}, & n \neq 0, \\ a^{\cos ^{2} \theta} b^{\sin ^{2} \theta}, & n=0 .\end{cases}
$$

Then the class of quasi-arithmetic mean [1] is defined by

$$
M_{p, n}(a, b)=p^{-1}\left(\frac{1}{2 \pi} \int_{0}^{2 \pi} p\left(r_{n}(\theta)\right) d \theta\right)
$$

(c) The Author(s) 2019. This article is distributed under the terms of the Creative Commons Attribution 4.0 International License (http://creativecommons.org/licenses/by/4.0/), which permits unrestricted use, distribution, and reproduction in any medium, provided you give appropriate credit to the original author(s) and the source, provide a link to the Creative Commons license, and indicate if changes were made. 


$$
=p^{-1}\left(\frac{2}{\pi} \int_{0}^{\pi / 2} p\left(r_{n}(\theta)\right) d \theta\right)
$$

where $p^{-1}$ is the inverse function of $p$.

Many important means are the special cases of the quasi-arithmetic mean $M_{p, n}(a, b)$. For example, from (1.1) and (1.2) we clearly see that

$$
M_{1 / x, 2}(a, b)=\frac{\pi}{2 \int_{0}^{\pi / 2}\left(a^{2} \cos ^{2} \theta+b^{2} \sin ^{2} \theta\right)^{-1 / 2} d \theta}=\operatorname{AGM}(a, b)
$$

is the Gaussian arithmetic-geometric mean [2-9], which is related to the complete elliptic integral of the first kind $\mathcal{K}=\mathcal{K}(r)=\int_{0}^{\pi / 2}\left(1-r^{2} \sin ^{2} \theta\right)^{-1 / 2} d \theta(0<r<1)$,

$$
T(a, b)=M_{x, 2}(a, b)=\frac{2}{\pi} \int_{0}^{\pi / 2} \sqrt{a^{2} \cos ^{2} \theta+b^{2} \sin ^{2} \theta} d \theta
$$

is the Toader mean [10-12], which can be expressed in terms of the complete elliptic integral of the second kind $\mathcal{E}=\mathcal{E}(r)=\int_{0}^{\pi / 2} \sqrt{1-r^{2} \sin ^{2} \theta} d \theta(0<r<1)$, and

$$
T Q(a, b)=M_{x, 0}(a, b)=\frac{\pi}{2} \int_{0}^{\pi / 2} a^{\cos ^{2} \theta} b^{\sin ^{2} \theta} d \theta
$$

is the Toader-Qi mean [13-15], which is related to the modified Bessel function of the first kind $I_{0}(x)=\sum_{n=0}^{\infty}(x / 2)^{2 n} /(n !)^{2}(x>0)$.

It is well-known that $\mathcal{K}(r)$ is strictly increasing from $(0,1)$ onto $(\pi / 2, \infty)$ and $\mathcal{E}(r)$ is strictly decreasing from $(0,1)$ onto $(1, \pi / 2)$. Moreover, $\mathcal{K}(r)$ and $\mathcal{E}(r)$ satisfy the following Landen identities and derivative formulas (see [16, Appendix E, pp. 474-475])

$$
\begin{aligned}
& \mathcal{K}\left(\frac{2 \sqrt{r}}{1+r}\right)=(1+r) \mathcal{K}, \quad \mathcal{E}\left(\frac{2 \sqrt{r}}{1+r}\right)=\frac{2 \mathcal{E}-r^{\prime 2} \mathcal{K}}{1+r}, \\
& \frac{d \mathcal{K}}{d r}=\frac{\mathcal{E}-r^{\prime 2} \mathcal{K}}{r r^{\prime 2}}, \quad \frac{d \mathcal{E}}{d r}=\frac{\mathcal{E}-\mathcal{K}}{r}, \\
& \frac{d\left(\mathcal{E}-r^{\prime 2} \mathcal{K}\right)}{d r}=r \mathcal{K}, \quad \frac{d(\mathcal{K}-\mathcal{E})}{d r}=\frac{r \mathcal{E}}{r^{\prime 2}} .
\end{aligned}
$$

In particular, $\mathcal{K}(r)$ and $\mathcal{E}(r)$ are the special cases of the Gaussian hypergeometric function [17-26] as follows:

$$
\mathcal{K}(r)=\frac{\pi}{2} F\left(\frac{1}{2}, \frac{1}{2} ; 1 ; r^{2}\right), \quad \mathcal{E}(r)=\frac{\pi}{2} F\left(-\frac{1}{2}, \frac{1}{2} ; 1 ; r^{2}\right),
$$

and the Gaussian hypergeometric function $F(a, b ; c ; x)$ with real parameters $a, b$, and $c(c \neq$ $0,-1,-2, \ldots)$ is defined by

$$
F(a, b ; c ; x)={ }_{2} F_{1}(a, b ; c ; x)=\sum_{n=0}^{\infty} \frac{(a, n)(b, n)}{(c, n)} \frac{x^{n}}{n !}
$$

for $x \in(-1,1)$, where $(a)_{0}=1$ for $a \neq 0,(a)_{n}=a(a+1)(a+2) \cdots(a+n-1)=\Gamma(a+n) / \Gamma(a)$ is the shifted factorial function and $\Gamma(x)=\int_{0}^{\infty} t^{x-1} e^{-t} d t(x>0)$ is the classical gamma function [27-35]. 
Recently, the bounds for the complete elliptic integrals have attracted the attention of many researchers. In particular, many remarkable inequalities and properties for $\mathcal{K}(r), \mathcal{E}(r)$ and $F(a, b ; c ; x)$ can be found in the literature [36-66].

In this article, we focus on the special quasi-arithmetic mean $E(a, b)$ obtained by substituting $p=\sqrt{x}$ and $n=1$ into (1.2), more explicitly,

$$
E(a, b)=M_{\sqrt{x}, 1}(a, b)=\left(\frac{2}{\pi} \int_{0}^{\pi / 2} \sqrt{a \cos ^{2} \theta+b \sin ^{2} \theta} d \theta\right)^{2},
$$

which can be rewritten in terms of complete elliptic integral of the second kind as

$$
E(a, b)= \begin{cases}\frac{4 a \mathcal{E}(\sqrt{1-b / a})^{2}}{\pi^{2}}, & a \geq b, \\ \frac{4 b \mathcal{E}(\sqrt{1-a / b})^{2}}{\pi^{2}}, & a<b .\end{cases}
$$

Very recently, Meng [67], and Yuan, Yu and Wang [68] proved that the double inequalities

$$
\begin{aligned}
& \lambda_{1} A(a, b)+\left(1-\lambda_{1}\right) G(a, b)<E(a, b)<\mu_{1} A(a, b)+\left(1-\mu_{1}\right) G(a, b), \\
& \lambda_{2} C(a, b)+\left(1-\lambda_{2}\right) H(a, b)<E(a, b)<\mu_{2} C(a, b)+\left(1-\mu_{2}\right) H(a, b)
\end{aligned}
$$

hold for $a, b>0$ with $a \neq b$ if and only if $\lambda_{1} \leq 3 / 4, \mu_{1} \geq 8 / \pi^{2}, \lambda_{2} \leq 4 / \pi^{2}$ and $\mu_{2} \geq 7 / 16$, where $A(a, b)=(a+b) / 2, G(a, b)=\sqrt{a b}, H(a, b)=2 a b /(a+b)$ and $C(a, b)=\left(a^{2}+b^{2}\right) /(a+b)$ are the arithmetic, geometric, harmonic and contra-harmonic means of $a$ and $b$, respectively.

Qian and Chu [69] showed that the double inequality

$$
\begin{aligned}
G^{p} & {[\lambda a+(1-\lambda) b, \lambda b+(1-\lambda) a] A^{1-p}(a, b) } \\
& <E(a, b) \\
& <G^{p}[\mu a+(1-\mu) b, \mu b+(1-\mu) a] A^{1-p}(a, b)
\end{aligned}
$$

holds for any $p \in[1, \infty)$ and all $a, b>0$ with $a \neq b$ if and only if $\lambda \leq 1 / 2-\sqrt{1-(2 \sqrt{2} / \pi)^{4 / p}} / 2$ and $\mu \geq 1 / 2-\sqrt{p} /(4 p)$.

From (1.7) and (1.8) we clearly see that

$$
\frac{3 A(a, b)}{4}+\frac{G(a, b)}{4}<E(a, b)<\frac{7 C(a, b)}{16}+\frac{9 H(a, b)}{16}
$$

for $a, b>0$ with $a \neq b$.

We define

$$
M_{1}(a, b)=\frac{3 A(a, b)+G(a, b)}{4}, \quad M_{2}(a, b)=\frac{7 C(a, b)+9 H(a, b)}{16} .
$$

Motivated by inequality (1.9), it is natural to ask what are the best possible parameters $\alpha_{i}, \beta_{i} \in(0,1)(i=1,2)$ such that the double inequalities

$$
\alpha_{1} M_{2}(a, b)+\left(1-\alpha_{1}\right) M_{1}(a, b)<E(a, b)<\beta_{1} M_{2}(a, b)+\left(1-\beta_{1}\right) M_{1}(a, b),
$$




$$
M_{2}(a, b)^{\alpha_{2}} M_{1}(a, b)^{1-\alpha_{2}}<E(a, b)<M_{2}(a, b)^{\beta_{2}} M_{1}(a, b)^{1-\beta_{2}}
$$

hold for all $a, b>0$ with $a \neq b$ ? The main purpose of this article is to answer this question.

\section{Lemmas}

In order to prove our main results we need several lemmas, which we present in this section.

Lemma 2.1 (See [16, Theorem 1.25]) Let $-\infty<a<b<\infty, f, g:[a, b] \rightarrow \mathbb{R}$ be continuous on $[a, b]$ and differentiable on $(a, b)$, and $g^{\prime}(x) \neq 0$ on $(a, b)$. If $f^{\prime}(x) / g^{\prime}(x)$ is increasing (decreasing) on $(a, b)$, then so are the functions

$$
\frac{f(x)-f(a)}{g(x)-g(a)} \text { and } \frac{f(x)-f(b)}{g(x)-g(b)} \text {. }
$$

If $f^{\prime}(x) / g^{\prime}(x)$ is strictly monotone, then the monotonicity in the conclusion is also strict.

Lemma 2.2 (See [70]) Suppose that the power series $f(x)=\sum_{n=0}^{\infty} a_{n} x^{n}$ and $g(x)=\sum_{n=0}^{\infty} b_{n} x^{n}$ have the radius of convergence $r>0$ with $b_{n}>0$ for all $n \in\{0,1,2, \ldots\}$. If the non-constant sequence $\left\{a_{n} / b_{n}\right\}_{n=0}^{\infty}$ is increasing (decreasing) for all $n>0$, then $f(x) / g(x)$ is strictly increasing (decreasing) on $(0, r)$.

Lemma 2.3 The following assertions hold true:

(1) The function $r \rightarrow\left(\mathcal{E}-r^{\prime 2} \mathcal{K}\right) / r^{2}$ is strictly increasing from $(0,1)$ onto $(\pi / 4,1)$;

(2) The function $r \rightarrow 2 \mathcal{E}-r^{\prime 2} \mathcal{K}$ is strictly increasing from $(0,1)$ onto $(\pi / 2,2)$;

(3) The function $r \rightarrow\left[\mathcal{K}-\mathcal{E}-\left(\mathcal{E}-r^{\prime 2} \mathcal{K}\right)\right] / r^{4}$ is strictly increasing from $(0,1)$ onto $(\pi / 16,+\infty)$.

Proof Parts (1) and (2) can be found in the literature [16, Theorem 3.21(1) and Exercise 3.43(13)]

For part (3), we clearly see that

$$
\frac{\mathcal{K}-\mathcal{E}-\left(\mathcal{E}-r^{\prime 2} \mathcal{K}\right)}{r^{4}}=\frac{\mathcal{K}-\mathcal{E}-\left(\mathcal{E}-r^{\prime 2} \mathcal{K}\right)}{\left(\mathcal{E}-r^{\prime 2} \mathcal{K}\right)^{2}} \cdot\left(\frac{\mathcal{E}-r^{\prime 2} \mathcal{K}}{r^{2}}\right)^{2}
$$

Therefore, part (3) follows easily from part (1) and [16, Exercise 3.43(25)].

Lemma 2.4 The function

$$
f(r)=\frac{8 / \pi^{2}\left(1+r^{2}\right)\left(2 \mathcal{E}-r^{\prime 2} \mathcal{K}\right)^{2}-\left(r^{2}+1\right)\left(r^{2}+2\right)}{r^{4}}
$$

is strictly increasing from $(0,1)$ onto $\left(3 / 16,64 / \pi^{2}-6\right)$.

Proof Let $f_{1}(r)=8 / \pi^{2}\left(1+r^{2}\right)\left(2 \mathcal{E}-r^{\prime 2} \mathcal{K}\right)^{2}-\left(r^{2}+1\right)\left(r^{2}+2\right)$ and $f_{2}(r)=r^{4}$, then $f_{1}\left(0^{+}\right)=$ $f_{2}\left(0^{+}\right)=0$ and $f(r)=f_{1}(r) / f_{2}(r)$.

A simple calculation yields

$$
\frac{f_{1}^{\prime}(r)}{f_{2}^{\prime}(r)}=\frac{f_{11}(r)}{f_{22}(r)}
$$


where

$$
\begin{aligned}
& f_{11}(r)=16\left(2 \mathcal{E}-r^{\prime 2} \mathcal{K}\right)^{2}+16\left(1+r^{2}\right)\left(2 \mathcal{E}-r^{\prime 2} \mathcal{K}\right)\left(\mathcal{E}-r^{\prime 2} \mathcal{K}\right) / r^{2}-\left(4 r^{2}+6\right), \\
& f_{22}(r)=4 r^{2}
\end{aligned}
$$

Moreover,

$$
\begin{aligned}
f_{11}\left(0^{+}\right)= & f_{22}\left(0^{+}\right)=0, \\
\frac{f_{11}^{\prime}(r)}{f_{22}^{\prime}(r)}= & 8\left(2 \mathcal{E}-r^{\prime 2} \mathcal{K}\right) \frac{\mathcal{E}-r^{\prime 2} \mathcal{K}}{r^{2}}+2\left(1+r^{2}\right)\left(\frac{\mathcal{E}-r^{\prime 2} \mathcal{K}}{r^{2}}\right)^{2} \\
& +2\left(1+r^{2}\right)\left(2 \mathcal{E}-r^{\prime 2} \mathcal{K}\right) \frac{\mathcal{K}-\mathcal{E}-\left(\mathcal{E}-r^{\prime 2} \mathcal{K}\right)}{r^{4}}-1 .
\end{aligned}
$$

From Lemma 2.3 and $(2.3)$, we clearly see that $f_{11}^{\prime}(r) / f_{22}^{\prime}(r)$ is strictly increasing on $(0,1)$. Equations (2.1)-(2.2) and Lemma 2.1 lead to the conclusion that $f(r)$ is strictly increasing on $(0,1)$.

Therefore, Lemma 2.4 follows from the monotonicity of $f(r)$, together with the facts that $f\left(0^{+}\right)=3 / 16$ and $f\left(1^{-}\right)=64 / \pi^{2}-6$.

\section{Lemma 2.5 The function}

$$
g(r)=\frac{\left(2 r^{6}+5 r^{4}+5 r^{2}+2\right)\left[2\left(\mathcal{E}-r^{\prime 2} \mathcal{K}\right)-r^{2} \mathcal{E}\right]}{r^{4}\left(3 r^{2}+4\right)\left(2 \mathcal{E}-r^{\prime 2} \mathcal{K}\right)}
$$

is strictly increasing from $(0,1)$ onto $(3 / 16,1)$.

Proof Let $g_{1}(r)=\left(2 r^{6}+5 r^{4}+5 r^{2}+2\right)\left[2\left(\mathcal{E}-r^{\prime 2} \mathcal{K}\right)-r^{2} \mathcal{E}\right]$ and $g_{2}(r)=r^{4}\left(3 r^{2}+4\right)\left(2 \mathcal{E}-r^{\prime 2} \mathcal{K}\right)$, then $g(r)=g_{1}(r) / g_{2}(r)$.

Making use of (1.3) and (1.4), we get

$$
\begin{aligned}
& \frac{2}{\pi}\left[2\left(\mathcal{E}-r^{\prime 2} \mathcal{K}\right)-r^{2} \mathcal{E}\right]=\sum_{n=0}^{\infty} \frac{3\left(\frac{1}{2}, n\right)\left(\frac{1}{2}, n+1\right)}{2 n !(n+2) !} r^{2 n+4}, \\
& \frac{2}{\pi}\left(2 \mathcal{E}-r^{\prime 2} \mathcal{K}\right)=1+\sum_{n=0}^{\infty} \frac{\left(\frac{1}{2}, n\right)^{2}}{4[(n+1) !]^{2}} r^{2 n+2} .
\end{aligned}
$$

It follows from (2.4) and (2.5) that

$$
\begin{aligned}
\frac{2}{\pi} g_{1}(r)= & \left(2 r^{6}+5 r^{4}+5 r^{2}+2\right) \sum_{n=0}^{\infty} \frac{3\left(\frac{1}{2}, n\right)\left(\frac{1}{2}, n+1\right)}{2 n !(n+2) !} r^{2 n+4} \\
= & \sum_{n=0}^{\infty} \frac{3\left(\frac{1}{2}, n\right)\left(\frac{1}{2}, n+1\right)}{n !(n+2) !} r^{2 n+4}+\sum_{n=0}^{\infty} \frac{15\left(\frac{1}{2}, n\right)\left(\frac{1}{2}, n+1\right)}{2 n !(n+2) !} r^{2 n+6} \\
& +\sum_{n=0}^{\infty} \frac{15\left(\frac{1}{2}, n\right)\left(\frac{1}{2}, n+1\right)}{2 n !(n+2) !} r^{2 n+8}+\sum_{n=0}^{\infty} \frac{3\left(\frac{1}{2}, n\right)\left(\frac{1}{2}, n+1\right)}{n !(n+2) !} r^{2 n+10} \\
= & r^{4}\left(\frac{3}{4}+\frac{33}{16} r^{2}+\frac{1245}{512} r^{4}+\sum_{n=0}^{\infty} \widetilde{A}_{n} r^{2 n+6}\right)
\end{aligned}
$$




$$
=r^{4} \sum_{n=0}^{\infty} A_{n} r^{2 n}
$$

and

$$
\begin{aligned}
\frac{2}{\pi} g_{2}(r) & =r^{4}\left(3 r^{2}+4\right)\left(1+\sum_{n=0}^{\infty} \frac{\left(\frac{1}{2}, n\right)^{2}}{4[(n+1) !]^{2}} r^{2 n+2}\right) \\
& =r^{4}\left(4+3 r^{2}+\sum_{n=0}^{\infty} \frac{\left(\frac{1}{2}, n\right)^{2}}{[(n+1) !]^{2}} r^{2 n+2}+\sum_{n=0}^{\infty} \frac{3\left(\frac{1}{2}, n\right)^{2}}{4[(n+1) !]^{2}} r^{2 n+4}\right) \\
& =r^{4}\left(4+4 r^{2}+\frac{13}{16} r^{4}+\sum_{n=0}^{\infty} \widetilde{B}_{n} r^{2 n+6}\right) \\
& =r^{4} \sum_{n=0}^{\infty} B_{n} r^{2 n},
\end{aligned}
$$

where

$$
\begin{aligned}
& A_{0}=\frac{3}{4}, \quad A_{1}=\frac{33}{16}, \quad A_{2}=\frac{1245}{512}, \quad A_{n}=\widetilde{A}_{n-3} \quad(n \geq 3), \\
& B_{0}=4, \quad B_{1}=4, \quad B_{2}=\frac{13}{16}, \quad B_{n}=\widetilde{B}_{n-3} \quad(n \geq 3), \\
& \widetilde{A}_{n}=\frac{3\left(\frac{1}{2}, n\right)\left(\frac{1}{2}, n+1\right)}{64(n+3) !(n+5) !}\left(45,765+152,928 n+192,838 n^{2}\right. \\
& \left.\quad+120,672 n^{3}+40,024 n^{4}+6720 n^{5}+448 n^{6}\right), \\
& \widetilde{B}_{n}=\frac{\left(\frac{1}{2}, n+1\right)^{2}\left(7 n^{2}+30 n+36\right)}{[4(n+3) !]^{2}}
\end{aligned}
$$

for $n \geq 0$.

It follows from (2.6) and (2.7) that

$$
g(r)=\frac{\sum_{n=0}^{\infty} A_{n} r^{2 n}}{\sum_{n=0}^{\infty} B_{n} r^{2 n}}
$$

for $r \in(0,1)$.

In order to prove the monotonicity of $g(r)$, Lemma 2.2 and (2.8) enable us to conclude that it suffices to show the monotonicity of $\left\{A_{n} / B_{n}\right\}_{n=0}^{\infty}$.

A simple calculation leads to

$$
\frac{A_{0}}{B_{0}}=\frac{3}{16}, \quad \frac{A_{1}}{B_{1}}=\frac{33}{64}, \quad \frac{A_{2}}{B_{2}}=\frac{1245}{416}, \quad \frac{A_{3}}{B_{3}}=\frac{3051}{128}
$$

and

$$
\begin{aligned}
\frac{A_{n+3}}{B_{n+3}}=\frac{\widetilde{A}_{n}}{\widetilde{B}_{n}}= & \frac{3}{8(n+4)(n+5)(2 n+1)\left(36+30 n+7 n^{2}\right)}(45,765+152,928 n \\
& \left.+192,838 n^{2}+120,672 n^{3}+40,024 n^{4}+6720 n^{5}+448 n^{6}\right),
\end{aligned}
$$




$$
\frac{\widetilde{A}_{n+1}}{\widetilde{B}_{n+1}}-\frac{\widetilde{A}_{n}}{\widetilde{B}_{n}}=\frac{3 \Delta_{1}(n)}{8 \Delta_{2}(n)}>0
$$

for $n \geq 0$, where

$$
\begin{aligned}
\Delta_{1}(n)= & 20,417,670+119,034,009 n+234,552,870 n^{2} \\
& +238,084,434 n^{3}+144,127,820 n^{4} \\
& +55,145,420 n^{5}+13,474,832 n^{6}+2,036,720 n^{7}+172,928 n^{8}+6272 n^{9}, \\
\Delta_{2}(n)= & (n+4)(n+5)(n+6)(2 n+1)(2 n+3)\left(36+30 n+7 n^{2}\right)\left(73+44 n+7 n^{2}\right) .
\end{aligned}
$$

It follows from Lemma 2.2 and $(2.8)-(2.10)$ that $g(r)$ is strictly increasing on $(0,1)$. Therefore, Lemma 2.5 follows easily from the monotonicity of $g(r)$, together with the facts that $g\left(0^{+}\right)=A_{0} / B_{0}=3 / 16$ and $g\left(1^{-}\right)=1$.

\section{Main results}

Theorem 3.1 The double inequality

$$
\alpha_{1} M_{2}(a, b)+\left(1-\alpha_{1}\right) M_{1}(a, b)<E(a, b)<\beta_{1} M_{2}(a, b)+\left(1-\beta_{1}\right) M_{1}(a, b)
$$

holds for $a, b>0$ with $a \neq b$ if and only if $\alpha_{1} \leq 3 / 16$ and $\beta_{1} \geq 64 / \pi^{2}-6$.

Proof Since $M_{1}(a, b), M_{2}(a, b)$ and $E(a, b)$ are symmetric and homogeneous of degree one, without loss of generality, we assume that $a>b>0$. Let $r=(1-\sqrt{b / a}) /(1+\sqrt{b / a}) \in(0,1)$, then (1.6) and (1.10), together with Landen identities, lead to

$$
\begin{aligned}
& E(a, b)=A(a, b) \frac{4(1+r)^{2}}{\pi^{2}\left(1+r^{2}\right)} \mathcal{E}^{2}\left(\frac{2 \sqrt{r}}{1+r}\right)=A(a, b) \frac{4}{\pi^{2}} \frac{\left(2 \mathcal{E}-r^{\prime 2} \mathcal{K}\right)^{2}}{1+r^{2}}, \\
& M_{1}(a, b)=A(a, b) \frac{r^{2}+2}{2\left(1+r^{2}\right)}, \quad M_{2}(a, b)=A(a, b) \frac{2+3 r^{2}+2 r^{4}}{2\left(1+r^{2}\right)^{2}}
\end{aligned}
$$

and

$$
\begin{aligned}
& E(a, b)-p M_{2}(a, b)-(1-p) M_{1}(a, b) \\
& \quad=A(a, b)\left[\frac{4}{\pi^{2}} \frac{\left(2 \mathcal{E}-r^{\prime 2} \mathcal{K}\right)^{2}}{1+r^{2}}-p \frac{2+3 r^{2}+2 r^{4}}{2\left(1+r^{2}\right)^{2}}-(1-p) \frac{r^{2}+2}{2\left(1+r^{2}\right)}\right] \\
& \quad=\frac{A(a, b) r^{4}}{2\left(1+r^{2}\right)^{2}}[f(r)-p],
\end{aligned}
$$

where $f(r)$ is defined as in Lemma 2.4.

Therefore, Theorem 3.1 follows from Lemma 2.4 and (3.3) immediately.

Theorem 3.2 The double inequality

$$
M_{2}(a, b)^{\alpha_{2}} M_{1}(a, b)^{1-\alpha_{2}}<E(a, b)<M_{2}(a, b)^{\beta_{2}} M_{1}(a, b)^{1-\beta_{2}}
$$

holds for $a, b>0$ with $a \neq b$ if and only if $\alpha_{2} \leq 3 / 16$ and $\beta_{2} \geq \log \left[32 /\left(3 \pi^{2}\right)\right] / \log (7 / 6)$. 
Proof Without loss of generality, we may assume that $a>b>0$. Let $r=(1-\sqrt{b / a}) /(1+$ $\sqrt{b / a}) \in(0,1)$, then (3.1) and (3.2) lead to

$$
\begin{aligned}
& \log E(a, b)-\lambda \log M_{2}(a, b)-(1-\lambda) \log M_{1}(a, b) \\
& \quad=\log \frac{8}{\pi^{2}}+\log \frac{\left(2 \mathcal{E}-r^{\prime 2} \mathcal{K}\right)^{2}}{r^{2}+2}-\lambda \log \frac{2 r^{4}+3 r^{2}+2}{\left(r^{2}+1\right)\left(r^{2}+2\right)} \\
& \triangleq \varphi(r) .
\end{aligned}
$$

Elaborated computations lead to

$$
\begin{aligned}
& \varphi(0)=0, \quad \varphi(1)=\log \frac{32}{3 \pi^{2}}-\lambda \log \frac{7}{6}, \\
& \varphi^{\prime}(r)=\frac{2 r\left(3 r^{2}+4\right)}{\left(r^{2}+1\right)\left(r^{2}+2\right)\left(2 r^{4}+3 r^{2}+2\right)}[g(r)-\lambda],
\end{aligned}
$$

where $g(r)$ is defined as in Lemma 2.5.

We divide the proof into three cases.

Case $1 . \lambda_{1}=3 / 16$. We clearly see from Lemma 2.5 that

$$
g(r)>\lambda_{1}
$$

for $r \in(0,1)$. It follows from (3.5)-(3.7) that $\varphi(r)>0$ for $r \in(0,1)$. This, in conjunction with (3.4), yields

$$
E(a, b)>M_{2}(a, b)^{\lambda_{1}} M_{1}(a, b)^{1-\lambda_{1}}
$$

for all $a, b>0$ with $a \neq b$.

Case 2. $\lambda_{2}=\log \left[32 /\left(3 \pi^{2}\right)\right] / \log (7 / 6)$. It follows from Lemma 2.5 that there exists $\delta \in(0,1)$ such that $g(r)<\lambda_{2}$ for $r \in(0, \delta)$ and $g(r)>\lambda_{2}$ for $r \in(\delta, 1)$. This, in conjunction with (3.6), implies that $\varphi(r)$ is strictly decreasing on $(0, \delta)$ and is strictly increasing on $(\delta, 1)$. Moreover, we clearly see from (3.5) that

$$
\varphi(0)=\varphi(1)=0
$$

The piecewise monotonicity property of $g(r)$ and (3.8) lead to the conclusion that $\varphi(r)<0$ for $r \in(0,1)$. Therefore,

$$
E(a, b)<M_{2}(a, b)^{\lambda_{2}} M_{1}(a, b)^{1-\lambda_{2}}
$$

for all $a, b>0$ with $a \neq b$ follows from (3.4).

Case $3.3 / 16<\lambda_{3}<\log \left[32 /\left(3 \pi^{2}\right)\right] / \log (7 / 6)$. By the locally sign-preserving property of limit, Lemma 2.5 and (3.6) enable us to know that there exists $\tau_{1} \in(0,1)$ such that $\varphi(r)$ is strictly decreasing on $\left(0, \tau_{1}\right)$. This, in conjunction with (3.5), implies that $\varphi(r)<0$ for 
$0<r<\tau_{1}$. Therefore,

$$
E(a, b)<M_{2}(a, b)^{\lambda_{3}} M_{1}(a, b)^{1-\lambda_{3}}
$$

for $b<a<\left[\left(1+\tau_{1}\right) /\left(1-\tau_{1}\right)\right]^{2} b$ follows from (3.4).

On the other hand, we clearly see from $(3.5)$ that $\varphi(1)>0$. This, in conjunction with the continuity of $\varphi(r)$, implies that there exists $\tau_{2} \in(0,1)$ such that $\varphi(r)>0$ for $\tau_{2}<r<1$. Therefore, it follows from (3.4) that

$$
E(a, b)>M_{2}(a, b)^{\lambda_{3}} M_{1}(a, b)^{1-\lambda_{3}}
$$

for $a>\left[\left(1+\tau_{2}\right) /\left(1-\tau_{2}\right)\right]^{2} b$.

Let $a=1$ and $b=1-r^{2}=r^{\prime 2}$, then (1.6), and Theorems 3.1 and 3.2 give rise to Corollary 3.3 immediately.

\section{Corollary 3.3 The double inequalities}

$$
\begin{aligned}
& \frac{3\left(7+18 r^{\prime 2}+7 r^{\prime 4}\right)}{256\left(1+r^{\prime 2}\right)}+\frac{13\left(1+6 r^{\prime}+r^{\prime 2}\right)}{128} \\
& <\mathcal{E}(r) \\
& \quad<\frac{\left(64-6 \pi^{2}\right)\left(7+18 r^{\prime 2}+7 r^{\prime 4}\right)}{16 \pi^{2}\left(1+r^{\prime 2}\right)}+\frac{\left(7 \pi^{2}-64\right)\left(1+6 r^{\prime}+r^{\prime 2}\right)}{8 \pi^{2}}, \\
& {\left[\frac{7+18 r^{\prime 2}+7 r^{\prime 4}}{16\left(1+r^{\prime 2}\right)}\right]^{3 / 16}\left(\frac{1+6 r^{\prime}+r^{\prime 2}}{8}\right)^{13 / 16}} \\
& <\mathcal{E}(r) \\
& <\left[\frac{7+18 r^{\prime 2}+7 r^{\prime 4}}{16\left(1+r^{\prime 2}\right)}\right]^{\frac{\log 32 /\left(3 \pi^{2}\right)}{\log (7 / 6)}}\left(\frac{1+6 r^{\prime}+r^{\prime 2}}{8}\right)^{\frac{\log \left(7 \pi^{2} / 64\right)}{\log (7 / 6)}}
\end{aligned}
$$

hold for all $r \in(0,1)$.

\section{Results and discussion}

In this article, we find the best possible parameters $\alpha_{1}, \beta_{1}, \alpha_{2}$ and $\beta_{2}$ on the interval $(0,1)$ such that the double inequalities

$$
\begin{aligned}
\alpha_{1}[ & {\left[\frac{7 C(a, b)}{16}+\frac{9 H(a, b)}{16}\right]+\left(1-\alpha_{1}\right)\left[\frac{3 A(a, b)}{4}+\frac{G(a, b)}{4}\right] } \\
& <E(a, b) \\
& <\beta_{1}\left[\frac{7 C(a, b)}{16}+\frac{9 H(a, b)}{16}\right]+\left(1-\beta_{1}\right)\left[\frac{3 A(a, b)}{4}+\frac{G(a, b)}{4}\right], \\
& {\left[\frac{7 C(a, b)}{16}+\frac{9 H(a, b)}{16}\right]^{\alpha_{2}}\left[\frac{3 A(a, b)}{4}+\frac{G(a, b)}{4}\right]^{1-\alpha_{2}} } \\
& <E(a, b)
\end{aligned}
$$




$$
<\left[\frac{7 C(a, b)}{16}+\frac{9 H(a, b)}{16}\right]^{\beta_{2}}\left[\frac{3 A(a, b)}{4}+\frac{G(a, b)}{4}\right]^{1-\beta_{2}}
$$

hold for all $a, b>0$ with $a \neq b$. Our results improve and refine the results given in $[67,68]$.

\section{Conclusion}

We present several sharp bounds for the quasi-arithmetic mean in terms of the combination of harmonic, geometric, arithmetic and contra-harmonic means. Our approach may have further applications in the theory of bivariate means.

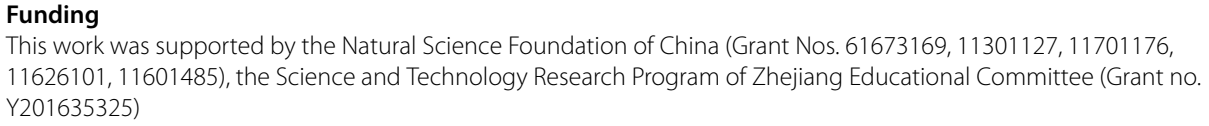

Competing interests

The authors declare that they have no competing interests.

\section{Authors' contributions}

All authors contributed equally to the writing of this paper. All authors read and approved the final manuscript.

\section{Author details}

${ }^{1}$ College of Science, Hunan City University, Yiyang, China. ${ }^{2}$ Department of Mathematics, Huzhou University, Huzhou, China.

\section{Publisher's Note}

Springer Nature remains neutral with regard to jurisdictional claims in published maps and institutional affiliations.

Received: 18 October 2018 Accepted: 8 February 2019 Published online: 20 February 2019

\section{References}

1. Toader, G.: Some mean values related to the arithmetic-geometric mean. J. Math. Anal. Appl. 218(2), 358-368 (1998)

2. Carlson, B.C., Vuorinen, M.: Inequality of the AGM and the logarithmic mean. SIAM Rev. 33(4), 653-654 (1991)

3. Qiu, S.-L., Vamanamurthy, M.K.: Sharp estimates for complete elliptic integrals. SIAM J. Math. Anal. 27(3), 823-834 (1996)

4. Alzer, H.: Sharp inequalities for the complete elliptic integral of the first kind. Math. Proc. Camb. Philos. Soc. 124(2), 309-314 (1998)

5. Anderson, G.D., Vamanamurthy, M.K., Vuorinen, M.: Functional inequalities for hypergeometric functions and complete elliptic integrals. SIAM J. Math. Anal. 23(2), 512-524 (1992)

6. Chu, Y.-M., Wang, M.-K.: Optimal inequalities between harmonic, geometric, logarithmic, and arithmetic-geometric means. J. Appl. Math. 2011, Article ID 618929 (2011)

7. Chu, Y.-M., Wang, M.-K.: Optimal Lehmer mean bounds for the Toader mean. Results Math. 61(3-4), 223-229 (2012)

8. Chu, Y.-M., Wang, M.-K.: Inequalities between arithmetic-geometric, Gini, and Toader means. Abstr. Appl. Anal. 2012, Article ID 830585 (2012)

9. Yang, Z.-H., Qian, W.-M., Chu, Y.-M., Zhang, W.: On approximating the arithmetic-geometric mean and complete elliptic integral of the first kind. J. Math. Anal. Appl. 462(2), 1714-1726 (2018)

10. Chu, Y.-M., Wang, M.-K., Qiu, S.-L., Qiu, Y.-F.: Sharp generalized Seiffert mean bounds for Toader mean. Abstr. Appl. Anal. 2011, Article ID 605259 (2011)

11. Chu, Y.-M., Wang, M.-K., Qiu, S.-L.: Optimal combinations bounds of root-square and arithmetic means for Toader mean. Proc. Indian Acad. Sci. Math. Sci. 122(1), 41-51 (2012)

12. Wang, J.-L., Qian, W.-M., He, Z.-Y., Chu, Y.-M.: On approximating the Toader mean by other bivariate means. J. Funct. Spaces 2019, Article ID 6082413 (2019)

13. Qi, F., Shi, X.-T., Liu, F.-F., Yang, Z.-H.: A double inequality for an integral mean in terms of the exponential and logarithmic means. Period. Math. Hung. 75(2), 180-189 (2017)

14. Qian, W.-M., Zhang, X.-H., Chu, Y.-M.: Sharp bounds for the Toader-Qi mean in terms of harmonic and geometric means. J. Math. Inequal. 11(1), 121-127 (2017)

15. Qi, F., Guo, B.-N.: Lévy-Khintchine representation of Toader-Qi mean. Math. Inequal. Appl. 21(2), 421-431 (2018)

16. Anderson, G.D., Vamanamurthy, M.K., Vuorinen, M.: Conformal Invariants, Inequalities, and Quasiconformal Maps. John Wiley \& Sons, New York (1997)

17. Yang, Z.-H., Chu, Y.-M., Wang, M.-K.: Monotonicity criterion for the quotient of power series with applications. J. Math. Anal. Appl. 428(1), 587-604 (2015)

18. Anderson, G.D., Qiu, S.-L., Vuorinen, M.: Precise estimates for differences of the Gaussian hypergeometric function. J. Math. Anal. Appl. 215(1), 212-234 (1997)

19. Ponnusamy, S., Vuorinen, M.: Univalence and convexity properties for Gaussian hypergeometric functions. Rocky Mt. J. Math. 31(1), 327-353 (2001) 
20. Wang, M.-K., Chu, Y.-M., Jiang, Y.-P.: Ramanujan's cubic transformation inequalities for zero-balanced hypergeometric functions. Rocky Mt. J. Math. 46(2), 679-691 (2016)

21. Wang, M.-K., Chu, Y.-M., Song, Y.-Q.: Asymptotical formulas for Gaussian and generalized hypergeometric functions. Appl. Math. Comput. 276, 44-60 (2016)

22. Wang, M.-K., Chu, Y.-M.: Refinements of transformation inequalities for zero-balanced hypergeometric functions. Acta Math. Sci. 37B(3), 607-622 (2017)

23. Wang, M.-K., Li, Y.-M., Chu, Y.-M.: Inequalities and infinite product formula for Ramanujan generalized modular equation function. Ramanujan J. 46(1), 189-200 (2018)

24. Wang, M.-K., Chu, Y.-M.: Landen inequalities for a class of hypergeometric functions with applications. Math. Inequal. Appl. 21(2), 521-537 (2018)

25. Wang, M.-K., Qiu, S.-L., Chu, Y.-M.: Infinite series formula for Hübner upper bound functions with applications to Hersch-Pfluger distortion function. Math. Inequal. Appl. 21(2), 629-648 (2018)

26. Zhao, T.-H., Wang, M.-K., Zhang, W., Chu, Y.-M.: Quadratic transformation inequalities for Gaussian hypergeometric function. J. Inequal. Appl. 2018, Article ID 251 (2018)

27. Maican, C.C.: Integral Evaluations Using the Gamma and Beta Functions and Elliptic Integrals in Engineering. International Press, Cambridge (2005)

28. Mortici, C.: New approximation formulas for evaluating the ratio of gamma functions. Math. Comput. Model. 52(1-2), 425-433 (2010)

29. Zhang, X.-M., Chu, Y.-M.: A double inequality for gamma function. J. Inequal. Appl. 2009, Article ID 503782 (2009)

30. Zhao, T.-H., Chu, Y.-M., Jiang, Y.-P.: Monotonic and logarithmically convex properties of a function involving gamma functions. J. Inequal. Appl. 2009, Article ID 728618 (2009)

31. Zhao, T.-H., Chu, Y.-M.: A class of logarithmically completely monotonic functions associated with a gamma function. J. Inequal. Appl. 2010, Article ID 392431 (2010)

32. Zhao, T.-H., Chu, Y.-M., Wang, H.: Logarithmically complete monotonicity properties relating to the gamma function. Abstr. Appl. Anal. 2010, Article ID 896483 (2010)

33. Yang, Z.-H., Qian, W.-M., Chu, Y.-M., Zhang, W.: On rational bounds for the gamma function. J. Inequal. Appl. 2017, Article ID 210 (2017)

34. Yang, Z.-H., Qian, W.-M., Chu, Y.-M., Zhang, W.: On approximating the error function. Math. Inequal. Appl. 21(2), 469-479 (2018)

35. Huang, T.-R., Han, B.-W., Ma, X.-Y., Chu, Y.-M.: Optimal bounds for the generalized Euler-Macheroni constant. J. Inequal. Appl. 2018, Article ID 118 (2018)

36. Anderson, G.D., Vamanamurthy, M.K., Vuorinen, M.: Functional inequalities for complete elliptic integrals and their ratios. SIAM J. Math. Anal. 21(2), 536-549 (1990)

37. Wang, M.-K., Chu, Y.-M., Zhang, W.: The precise estimates for the solution of Ramanujan's generalized modular equation. Ramanujan J. https://doi.org/10.1007/s11139-018-0130-8

38. Qiu, S.-L., Vamanamurthy, M.K., Vuorinen, M.: Some inequalities for the growth of elliptic integrals. SIAM J. Math. Anal. 29(5), 1224-1237 (1998)

39. Barnard, R.W., Pearce, K., Richards, K.C.: An inequality involving the generalized hypergeometric function and the arc length of an ellipse. SIAM J. Math. Anal. 31(3), 693-699 (2000)

40. Barnard, R.W., Pearce, K., Richards, K.C.: A monotonicity properties involving ${ }_{3} F_{2}$, and comparisons of the classical approximations of elliptical arc length. SIAM J. Math. Anal. 32(2), 403-419 (2000)

41. Qiu, S.-L., Ma, X.-Y., Chu, Y.-M.: Sharp Landen transformation inequalities for hypergeoemtric functions, with applications. J. Math. Anal. Appl. https://doi.org/10.1016/j.jmaa.2019.02.018

42. Yang, Z.-H., Qian, W.-M., Chu, Y.-M.: Monotonicity properties and bounds involving the complete elliptic integrals of the first kind. Math. Inequal. Appl. 21(4), 1185-1199 (2018)

43. Zhang, X.-H., Wang, G.-D., Chu, Y.-M.: Remarks on generalized elliptic integrals. Proc. R. Soc. Edinb., Sect. A 139(2), 417-426 (2009)

44. Zhang, X.-H., Wang, G.-D., Chu, Y.-M.: Convexity with respect to Hölder mean involving zero-balanced hypergeometric functions. J. Math. Anal. Appl. 353(1), 256-259 (2009)

45. András, S., Baricz, Á.: Bounds for complete elliptic integrals of the first kind. Expo. Math. 28(4), 357-364 (2010)

46. Neuman, E.: Inequalities and bounds for generalized complete integrals. J. Math. Anal. Appl. 373(1), 203-213 (2011)

47. Wang, M.-K., Chu, Y.-M., Qiu, Y.-F., Qiu, S.-L.: An optimal power mean inequality for the complete elliptic integrals. Appl. Math. Lett. 24(6), 887-890 (2011)

48. Chu, Y.-M., Wang, M.-K., Qiu, Y.-F.: On Alzer and Qiu's conjecture for complete elliptic integral and inverse hyperbolic tangent function. Abstr. Appl. Anal. 2011, Article ID 697547 (2011)

49. He, X.-H., Qian, W.-M., Xu, H.-Z., Chu, Y.-M.: Sharp power mean bounds for two Sándor-Yang means. Rev. R. Acad. Cienc. Exactas Fís. Nat., Ser. A Mat. https://doi.org/10.1007/s13398-019-00643-2

50. Bhayo, B.A., Vuorinen, M.: On generalized complete integrals and modular functions. Proc. Edinb. Math. Soc. (2) 55(3), 591-611 (2012)

51. Wang, M.-K., Qiu, S.-L., Chu, Y.-M., Jiang, Y.-P.: Generalized Hersch-Pfluger distortion function and complete elliptic integrals. J. Math. Anal. Appl. 385(1), 221-229 (2012)

52. Wang, M.-K., Chu, Y.-M., Qiu, S.-L., Jiang, Y.-P.: Convexity of the complete elliptic integrals of the first kind with respect to Hölder means. J. Math. Anal. Appl. 388(2), 1141-1146 (2012)

53. Chu, Y.-M., Wang, M.-K., Jiang, Y.-P., Qiu, S.-L.: Concavity of the complete elliptic integrals of the second kind with respect to Hölder means. J. Math. Anal. Appl. 395(2), 637-642 (2012)

54. Chu, Y.-M., Qiu, Y.-F., Wang, M.-K.: Hölder mean inequalities for complete elliptic integrals. Integral Transforms Spec. Funct. 23(7), 521-527 (2012)

55. Chu, Y.-M., Wang, M.-K., Qiu, S.-L., Jiang, Y.-P.: Bounds for complete elliptic integrals of the second kind with applications. Comput. Math. Appl. 63(7), 1177-1184 (2012)

56. Wang, M.-K., Chu, Y.-M.: Asymptotical bounds for complete elliptic integrals of the second kind. J. Math. Anal. Appl. 402(1), 119-126 (2013)

57. Chu, Y.-M., Wang, M.-K., Qiu, Y.-F., Ma, X.-Y.: Sharp two parameters bounds for the logarithmic mean and the arithmetic-geometric mean of Gauss. J. Math. Inequal. 7(3), 349-355 (2013) 
58. Wang, M.-K., Chu, Y.-M., Qiu, S.-L.: Some monotonicity properties of generalized elliptic integrals with applications. Math. Inequal. Appl. 16(3), 671-677 (2013)

59. Chu, Y.-M., Qiu, S.-L., Wang, M.-K.: Sharp inequalities involving the power mean and complete elliptic integral of the first kind. Rocky Mt. J. Math. 43(5), 1489-1496 (2013)

60. Wang, M.-K., Chu, Y.-M., Jiang, Y.-P., Qiu, S.-L.: Bounds of the perimeter of an ellipse using arithmetic, geometric and harmonic means. Math. Inequal. Appl. 17(1), 101-111 (2014)

61. Wang, G.-D., Zhang, X.-H., Chu, Y.-M.: A power mean inequality involving the complete elliptic integrals. Rocky Mt. J. Math. 44(5), 1661-1667 (2014)

62. Yang, Z.-H., Chu, Y.-M.: A monotonicity property involving the generalized elliptic integral of the first kind. Math. Inequal. Appl. 20(3), 729-735 (2017)

63. Yang, Z.-H., Chu, Y.-M., Zhang, W.: High accuracy asymptotic bounds for the complete elliptic integral of the second kind. Appl. Math. Comput. 348, 552-564 (2019)

64. Yang, Z.-H., Qian, W.-M., Chu, Y.-M., Zhang, W.: Monotonicity rule for the quotient of two functions and its application 2017 J. Inequal. Appl. Article ID 106 (2017)

65. Yang, Z.-H., Zhang, W., Chu, Y.-M.: Sharp Gautschi inequality for parameter $0<p<1$ with applications. Math. Inequal, Appl. 20(4), 1107-1120 (2017)

66. Huang, T.-R., Tan, S.-Y., Ma, X.-Y., Chu, Y.-M.: Monotonicity properties and bounds for the complete $p$-elliptic integrals. J. Inequal. Appl. 2018, Article ID 239 (2018)

67. Meng, M.-L.: Inequalities for a class of new arithmetic means. Thesis (B.S.), Huzhou, University (2017). (in Chinese)

68. Yuan, Q., Yu, F.-T., Wang, M.-K.: Inequalities for the complete elliptic integrals of the second kind in terms of means. J. Huzhou Univ. 39(2), 12-16 (2017)

69. Qian, W.-M., Chu, Y.-M.: Sharp bounds for a special quasi-arithmetic mean in terms of arithmetic and geometric means with two parameters. J. Inequal. Appl. 2017, Article ID 274 (2017)

70. Biernacki, M., Krzyż, J.: On the monotonicity of certain functionals in the theory of analytic functions. Ann. Univ. Mariae Curie-Skłodowska, Sect. A 9, 135-147 (1955)

\section{Submit your manuscript to a SpringerOpen ${ }^{\circ}$ journal and benefit from:}

- Convenient online submission

- Rigorous peer review

- Open access: articles freely available online

- High visibility within the field

Retaining the copyright to your article

Submit your next manuscript at $\gg$ springeropen.com 